\title{
Determinants of anti-retroviral regimen changes among HIV/AIDS patients of east and west Wollega zone health institutions, Oromia region, west Ethiopia: a cross-sectional study
}

\author{
Amsalu Bokore ${ }^{1^{*}}$, Belay Korme ${ }^{1}$ and Getu Bayisa ${ }^{2}$
}

\begin{abstract}
Background: Human Immunodeficiency Virus (HIV) is one of the main causes of morbidity and mortality; because of this it continues to be a major global public health concern. It has believed to kill more than 34 million lives so far. Sub Saharan Africa constitutes about 70\% of people living with HIV among the 37 million on the globe. This region, accounted for more than two third of the global new HIV infections and about 15 million (40\%) were receiving antiretroviral therapy (ART) at the end of 2014 throught the world. ART has fundamentally changed the treatment of HIV and transformed this infection from a disease of high mortality to chronic and medically managed disease. The issues of drug induced toxicities \& complexity of current highly active antiretroviral therapy (HAART) regimens has remained of great concern. The aim of this study was to determine factors leading to antiretroviral regimen changes among HIV/AIDS Patients in the study area.
\end{abstract}

Methods: A facility based retrospective cross-sectional study was conducted from April 28, 2017 to May 30, 2017 in the ART clinics of east and west Wollega zone health institutions using a pre-tested data collecting form and chart review. The sample included the 243 patients whose medication had been switched.

Results: Majority 145 (59.67\%) of the patients had been on ART for $>10$ years duration. More than half 126(51.9\%) of the patients had received tuberculosis (TB) treatment and almost three out of five patients (57.2\%) had received isoniazid \& cotrimoxazole prophylaxis. The most common reason for regimen change was peripheral neuropathy 146(60.1\%) and the most common medication for this reason was stavudine, lamivudine and neverapine based 108(44.44\%).

Conclusions: The number of patients who changed ARV drug in our resource constrained setting present a challenge to the restricted treatment choices that we currently own. Less toxic and better-tolerated HIV treatment options should be available and used more frequently.

Keywords: HIV/AIDS, HAART, ARV drug, Regimen change, Wollega

\footnotetext{
* Correspondence: bokore.amsalu@yahoo.com

${ }^{1}$ Nekemte referral hospital, Nekemte, Ethiopia

Full list of author information is available at the end of the article
}

(c) The Author(s). 2018 Open Access This article is distributed under the terms of the Creative Commons Attribution 4.0 International License (http://creativecommons.org/licenses/by/4.0/), which permits unrestricted use, distribution, and reproduction in any medium, provided you give appropriate credit to the original author(s) and the source, provide a link to the Creative Commons license, and indicate if changes were made. The Creative Commons Public Domain Dedication waiver (http://creativecommons.org/publicdomain/zero/1.0/) applies to the data made available in this article, unless otherwise stated. 


\section{Background}

Human immunodeficiency virus (HIV) annihilates and compromises the function of immune cells. Immunity of infected individuals gradually depletes and susceptibility to a wide range of infections and diseases would be boosted. Acquired immunodeficiency syndrome (AIDS) is the most advanced stage of HIV infection, which can take from 2 to 15 years to develop depending on the individual and it can be explained by the progression of opportunistic infections, or other intense clinical manifestations and certain cancers $[1,2]$.

HIV is one of the main causes of morbidity and mortality; because of this it continues to be a major global public health concern. It has believed to kill more than 34 million lives so far. Sub Saharan Africa constitutes about $70 \%$ of people living with HIV among the thirty seven million on the globe. This region, accounted for more than two third of the global new HIV infections in 2014 with only $12 \%$ of the global population. Globally people receiving ART were about fifteen million $(40 \%)$ among those living with HIV of which about fourteen million were in low- and middle -income countries and nearly one million were children [2].

Ethiopia is among the countries most affected by HIV/ AIDS with prevalence of $1.9 \%$ for women and $1.0 \%$ for men in 2011 [3] and it is also in a low generalized HIV epidemic with significant heterogeneity among regions and population groups [4]. The existence of HIV infection in Ethiopia was recognized in the early 1980s with the first two AIDS cases reported in 1986. The predominant strain is HIV-1 subtype C, predominantly spread through unprotected heterosexual intercourse [5]. Since 2000 the epidemic has declined [6] and recent figures show that HIV infection has significantly decreased over the years in the country [4].

Highly active antiretroviral therapy (HAART) has fundamentally changed the treatment of HIV and changed this infection from a disease of high mortality to chronic and medically managed disease which is a radical change in controlling the hardship of HIV/AIDS. However drug resistance and side effects were the great concern in these advancements [7]. Revolution in the care of patients with HIV/AIDS occurred due to the innovation of potent HAART in around 2000. The qualities of life of people living with HIV/AIDS (PLWHA) have improved and these treatments have dramatically reduced rates of mortality and morbidity among these patients. This result was also confirmed by World health organization (WHO) progress report. ARV drugs produce these effects by restoration of number and quality of cluster of difference (CD4) cells and suppressing viral replication [8].

The goal of ART is to attain maximal and durable suppression of the viral replication. Viral suppression enables recovery of the immune response and thereby reduces risk of opportunistic infections (OIs) and death [1].

The decision to initiate ART for adults and adolescents depend on: WHO stage 3 and 4 disease irrespective of CD4 cell count, CD4 count $\leq 500$ cells $/ \mathrm{mm}^{3}$ irrespective of WHO clinical stage and active tuberculosis (TB) co-infection with HIV irrespective of CD4 cell count according to standard treatment guideline of Ethiopia [6].

Triple combination therapy has been in use for more than two decades globally. Currently, the preferred first regimen triple therapy in Ethiopia consists of, two $\mathrm{Nu}$ cleoside Reverse Transcriptase Inhibitors (NRTIs) and one Protease Inhibitor (PI) or a Non-Nucleoside Reverse Transcriptase Inhibitor (NNRTI) or a triple therapy of three NRTIs. Based on the guideline, common ART regimens in the country are; Tenofovir (TDF)/ Emtricitabine (FTC)/ Efavirenz (EFV) or Nevirapine (NVP); alternatives are TDF/ Lamivudine (3TC) /EFV or NVP, Zidovudine (ZDV)/3TC/EFV or NVP. Other options are Abacavir $(\mathrm{ABC}) / 3 \mathrm{TC} / \mathrm{NVP}, \mathrm{ABC} / 3 \mathrm{TC} / \mathrm{EFV}$ and $A B C / 3 T C / Z D V$. The second line regimen consists of $\mathrm{ZDV} \pm 3 \mathrm{TC}+$ Lopinavir/ritonavir (LPV/r) (or Atazanavir/ritonavir $(\mathrm{ATV} / \mathrm{r})), \mathrm{ZDV}+\mathrm{ABC}+\mathrm{LPV} / \mathrm{r} \quad$ (or $\mathrm{ATV} / \mathrm{r}$ ), $\mathrm{TDF} / 3 \mathrm{TC} \pm \mathrm{ZDV}+\mathrm{LPV} / \mathrm{r}$ (or $\mathrm{ATV} / \mathrm{r}$ ), $\mathrm{ABC} /$ Didanosine (ddI) /LPV/r (or ATV/r), EFV or NVP / $\mathrm{LPV} / \mathrm{r}$ (or ATV/r) $[1,9]$.

Changes of multiple medications in HAART regimens were commonly required simultaneously. These changes may be due to co morbidity with other chronic diseases, a desire for pregnancy, poor adherence, stock out of drugs, treatment failure, long term toxicity or acute toxicity. The approaches to change ART regimens depend largely on amount of previous ART experience, available treatment options and reason for change. For example effective treatment can be accomplished by substituting another agent for the drug which has unpleasant effect in the regimen when it develops to certain drugs in the regimen $[7,10]$.

The issues of drug toxicities and complexity of current HAART regimens has remained of great concern despite ARTs being of much help to the health of HIV/AIDS patients. Suboptimal therapy, discontinuation, and treatment failure can be resulted from treatment toxicities and adherence problems [11]. The consequence of these may complicate the management and lead to toxicity, loss to follow-up, compromise the effectiveness of HAART regimens, drug interactions and drug resistance [12].

Knowledge of the determinants of ART change may help to minimize the risk factors. These benefits in decreasing the rate of regimen change, treatment failure, drug resistance, and improve the quality of life of the patient. Antiretroviral treatment change should be done 
when necessary to spare the future treatment options. The approach to patients who need to switch will differ depending on several issues, including ART experience and available options. Regimen substitution requires adjustment in learning the new medication about the treatment dosing, time of intake and deal with many individual based inconveniences, which might be challenging and reason for non-adherence [10, 13].

Data on modification of HAART and factors associated with ARV drug regimen change are limited among HIV/AIDS patients in Ethiopia. Most of the surveys used were on small sample of patients who were on ART for less than three years. As a result it is important to understand common reasons of ARV drug switch in patients on long period exposure to ART.

Therefore, this study attempts to investigate the major determinants of HAART regimen change among HIV/ AIDS patients in east and west Wollega zone health institutions by using cross-sectional study.

\section{Objective}

\section{General objective}

$\checkmark$ To assess determinants of antiretroviral regimen change among HIV/AIDS patients in east and west Wollega zone health institutions, Oromia region, west Ethiopia.

\section{Specific objectives}

$\checkmark$ To assess determinants of antiretroviral regimen change among HIV/AIDS positive patients.

$\checkmark$ To identify the pattern of initial ART regimens and the subsequent changes.

$\checkmark$ To assess the relationship between patient characteristics and reasons for initial ART change.

\section{Methods}

\section{Study area, design, and period}

The study was conducted in the ART clinics of east and west Wollega zone health institutions, Oromia region, west Ethiopia; Nekemte town which is the capital of east Wollega is located $328 \mathrm{~km}$ where as Gimbi town which is the capital of west Wollega is $438 \mathrm{Km}$ western to Addis Ababa [14].

The area is well known by its coffee production. The economy of the people is based on subsistence farming and livestock rearing. The climatic condition of the area is 'woinadega' (semi-desert) and it is found at $2080 \mathrm{~m}$ above sea level.

A facility based retrospective cross-sectional study was conducted by reviewing patient information sheets and physician diagnostic cards to assess reasons for HAART regimen change. The study was conducted from April 28, 2017 to May 30, 2017.

\section{Source and study population}

All HIV/AIDS positive patients who were greater than 18 years and on HAART in east and west Wollega zone health institutions ART Clinic from April 28, 2007 to April 28, 2017 were the source population. All HIV/ AIDS positive patients greater than 18 years who had undergone HAART regimen change in east and west Wollega zone health institutions ART Clinic in between April 28, 2007 to April 28, 2017 were the study population.

\section{Inclusion and exclusion criteria}

Inclusion criteria:

$\checkmark$ Patients on follow up in the ART clinic who had undergone HAART regimen change until the study period.

$\checkmark$ Patients on follow up in the ART clinic who were on second line regimen when the study was undergone.

$\checkmark$ HIV/AIDS patients who were greater than18 years.

$\checkmark$ Patients receiving HAART regimen for at least 6 months at the beginning of the study period

Exclusion criteria:

$\checkmark$ Patient information cards with incomplete information (Patient information card which had no one or more of information like information on demographics, WHO clinical stage, CD4 count, initiation regimen and changed regimen, duration of initial therapy, and causes for regimen change).

$\checkmark$ Patients with less than 6 months on HAART regimen.

$\checkmark$ Patients who didn't switch HAART regimen.

$\checkmark$ Under eighteen year old HIV/AIDS patients.

$\checkmark$ Deceased patients

$\checkmark$ Transfer out patients

\section{Sample population}

A total of 243 patients who had undergone HAART regimen change in the ART clinics of east and west Wollega zone health institutions from April 28, 2007 to April 28, 2017 were included in the study while patients below 18 years were excluded from the study. Patient information cards that showed a change in the initial treatment regimen were assessed and analyzed, to identify the common reasons that resulted in a change from the initial treatment regimen.

\section{Study variables}

Independent variables 
$\checkmark$ Socio-demographic characteristics: age at initiation, sex, marital status, educational status.

$\checkmark$ Disease related variables: baseline WHO stage, base line CD4, and baseline weight.

$\checkmark$ ART related variables: types of initial regimen

\section{Dependent variables}

\section{$\checkmark$ Reasons for change}

\section{Data procedure and management}

\section{Data collection procedure}

Data abstraction form was developed based on the objectives of the study. It contained socio-demographic, clinical information and ART information such as, CD4 count, WHO stage, initial regimen, date on which treatment was started, date of ARV drug switch, duration of initial ARV therapy before first switch, regimen switched to, and causes for regimen change. The types of toxicity and treatment failure reasons were included. If there was ARV drug switch for the second and third time it was recorded in a similar manner. For data collection four 10th grade completed students were recruited. One pharmacist and one druggist from each health institutions were also recruited as supervisors.

\section{Data collectors recruitment and training}

Data collectors were recruited and trained methods of data collection prior to the start of actual data collection.

\section{Data quality assurance/control}

Training was provided for supervisors and data collectors and they were standardized. Data abstraction form was pre-tested on randomly selected patient information cards to identify any drawbacks in Shambu hospital which is found in Horro Gudru Wollega zone before the actual survey and improvements were made. The principal investigator supervised the data collection. Every questionnaire was checked for completeness and logical consistency.

\section{Data processing and analysis}

The data were coded and entered in to a computer using statistical Package for the Social Sciences (SPSS) software for windows version 20 and the analysis was performed after the data were cleaned, edited and processed. Distribution of Patients such as percentages and their number by socio demographic characteristics and other relevant variables in the study were described using descriptive analysis.

\section{Ethical considerations}

An official letter was written by department of pharmacy, college of public health and medical sciences,
Wollega University to zonal and woreda health offices of east and west Wollega zone Administration to get permission. After permission to conduct the study was obtained, data has been collected in one of refilling rooms at ART Clinic by safe keeping of records.

Only numerical identifications were used as a reference, confidentiality and anonymity of subject was maintained by not recording and identifying details, such as name or any other personal details. No disclosure of any name of the patients, the healthcare provider or drug product was made in relation to the finding.

\section{Operational definitions}

ABC based regimen: regimen containing abacavir as one of the NRTI backbones and may have different NNRI or PI bases.

Antiretroviral drug switch/change: it is the change of one or two ARV drugs from the initial drug regimens.

AZT based regimen: regimen containing zidovudine as one of the NRTI backbones and may have different NNRI or PI bases.

Table 1 Socio-demographic characteristics of HIV/AIDS patients who changed their HAART regimen in east and west Wollega zone health institutions, April 28, 2007 to April 28, 2017

\begin{tabular}{ll}
\hline Demographic Characteristics & $\mathrm{N}(\%)$ \\
\hline Age in years & $19(7.8)$ \\
$20-34$ & $174(71.6)$ \\
$35-49$ & $50(20.6)$ \\
$\geq 50$ & \\
Sex & $127(52.3)$ \\
Female & $116(47.7)$ \\
Male & \\
Marital status & $18(7.4)$ \\
Single & $154(63.4)$ \\
Married & $12(4.9)$ \\
Divorced & $59(24.3)$ \\
Widowed & \\
Educational status & $74(30.5)$ \\
No formal education & $92(37.9)$ \\
Primary school education & $52(21.4)$ \\
Secondary school education & $25(10.3)$ \\
Higher institute education & \\
Family size & \\
Less than five & \\
5-10 & \\
Rrban & \\
\hline
\end{tabular}


Table 2 Clinical characteristics of HIV/AIDS patients who changed their HAART regimen in east and west Wollega zone health institutions, April 28, 2007 to April 28, 2017

\begin{tabular}{|c|c|c|c|c|}
\hline & & $\begin{array}{l}\text { On initiation } \\
\text { of ART }\end{array}$ & $\begin{array}{l}\text { Before ART } \\
\text { switch }\end{array}$ & $\begin{array}{l}\text { On data } \\
\text { collection }\end{array}$ \\
\hline \multirow[t]{5}{*}{ WHO clinical stage } & stage I & 17 & 60 & 237 \\
\hline & stage II & 40 & 55 & 6 \\
\hline & stage III & 180 & 126 & 0 \\
\hline & stage IV & 6 & 2 & 0 \\
\hline & Total & 243 & 243 & 243 \\
\hline \multirow[t]{4}{*}{ CD4 count (cells/ml) } & $<200$ & 180 & 42 & 27 \\
\hline & $200-350$ & 60 & 54 & 26 \\
\hline & $>350$ & 03 & 147 & 190 \\
\hline & Total & 243 & 243 & 243 \\
\hline \multirow[t]{4}{*}{ Weight (kg) } & $<45$ & 51 & 24 & 24 \\
\hline & $45-60$ & 164 & 155 & 137 \\
\hline & $>60$ & 28 & 64 & 82 \\
\hline & Total & 243 & 243 & 243 \\
\hline
\end{tabular}

Co morbidity: is defined as the occurrence of one or more additional disorders which are on drug therapy with HIV/AIDS simultaneously (TB, diabetes, hypertension).

d4T based regimen: regimen containing Stavudine as one of the NRTI backbones and may have different NNRI or PI bases.

TDF based regimen: regimen containing Tenofovir as one of the NRTI backbones and may have different NNRI or PI bases.

Toxicity: is defined as the occurrence of adverse events such as diarrhea, nausea, vomiting, anemia, rash, fatigue, peripheral neuropathy, lipodystrophy, metabolic disturbances, CNS abnormalities or any other unwanted effect related to HAART.

Transfer out: Patients who changed their follow up to other health institution.

\section{Results \\ Socio-demographic characteristics of patients whose ART regimen changed}

The mean age of patients was $43.68(\mathrm{SD} \pm 8.2)$ years. Majority of the patients $174(71.6 \%)$ were in the age range of $35-49$ years and more than half $127(52.3 \%)$ of the patients were females. About 154(63.4\%) of the patients were married. In this study only $77(31.7 \%)$ of the patients received greater than secondary school education. Regarding the family size $96(39.5 \%)$ of the patients had $<5$ family size whereas $61(25.1 \%)$ had $>10$ family size. Regarding place of residence 209(86\%) were urban dwellers where as $34(14 \%)$ were rural dwellers as shown in (Table 1).

\section{Clinical characteristics of patients whose ART regimen was changed}

During initiation of ART 180(74.1\%) of the patients were on WHO clinical stage III; whereas WHO stage after the ART switch was stage I for 237(97.53\%) of patients. Similarly more than two third $180(74.1 \%)$ of patients had baseline CD4 count less than 200 cells $/ \mu \mathrm{L}$ on initiation but most 190(78.19\%) of patients had $>350$ cells $/ \mu \mathrm{L}$ after ART switch. In addition, the weight of the majority 164(67.5\%) of patients during initiation of ART was between 45 and $60 \mathrm{~kg}$ (Table 2). Majority 145 (59.67\%) of the patients had been on ART for $>10$ years duration (Fig. 1). About $126(51.85 \%$ ) of the patients had received TB treatment whereas $117(48.15 \%)$ of the patients did not receive TB treatment. Regarding OI

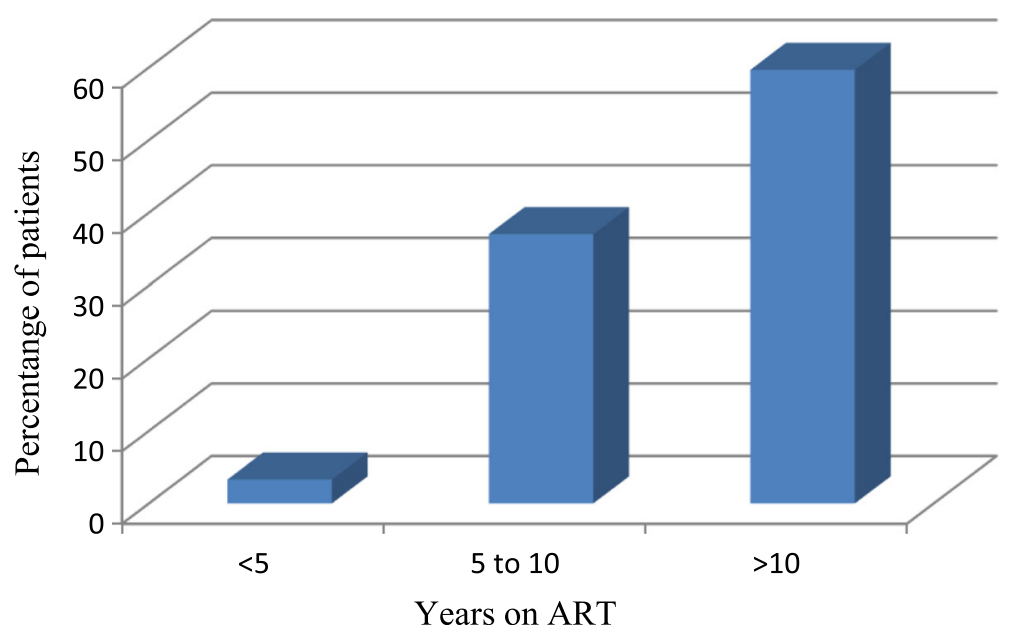

Fig. 1 Years of stay on ART of HIV/AIDS patients who changed their HAART regimen in east and west Wollega zone health institutions, April 28, 2007 to April 28, 2017 
Table 3 Ol prophylaxis taken by study population in east and west Wollega zone health institutions, April 28, 2007 to April 28, 2017

\begin{tabular}{lll}
\hline Type of Ol prophylaxis & Frequency & Percent \\
\hline Cotrimoxazole \& Isoniazid & 139 & 57.2 \\
Cotrimoxazole & 95 & 39.1 \\
Neither & 9 & 3.7 \\
Total & 243 & 100.0 \\
\hline
\end{tabular}

prophylaxis $139(57.2 \%)$ of the patients received Cotrimoxazole and Isonazid prophylaxis (Table 3 ).

\section{Patterns of initial ART regimen and regimen switched to/ changed regimen}

Majority of the patients $159(65.4 \%)$ started their initial ART on D4t-3TC-NVP regimens, followed by D4t-3TC-EFV 55 (22.6\%). Only $12 \%$ of patients started initial ART regimen on AZT based regimen (Table 4).

The HAART regimen of majority of the patients 168(69.14\%) was changed to TDF based regimen. Whereas about $69(28.4 \%)$ of the patients initial HAART regimen was changed to AZT based regimen and only $6(2.5 \%)$ patients initial HAART regimen was changed to $\mathrm{ABC}$ based regimen (Table 5).

\section{Reasons for ART change}

The main reason for antiretroviral regimen change was Peripheral neuropathy 146(60.1\%) followed by hepatotoxicity, d4t faith out, CNS toxicity, Anemia, Rash etc. (Table $4)$. The most common reason for regimen change was peripheral neuropathy $146(60.1 \%)$ and the most common medication for this reason was stavudine, lamivudine and neverapine based 108(44.44\%) (Table 6 and 7).

\section{Discussion}

The present retrospective cross sectional study of HIV/ AIDS patients who changed their HAART regimen in east and west Wollega zone health institutions described the pattern of ART regimen and the common reasons for ARV drug switch. Such studies would be helpful in understanding the complexity of ART use of patients in

Table 4 HAART regimen at initiation among HIV/AIDS patients who changed their HAART regimen in east and west Wollega zone health institutions, April 28, 2007 to April 28, 2017

\begin{tabular}{lll}
\hline Initial HAART regimen & Frequency & Percent \\
\hline D4t-3TC-NVP & 159 & 65.4 \\
D4t-3TC-EFV & 55 & 22.6 \\
AZT-3TC-NVP & 14 & 5.8 \\
AZT-3TC-EFV & 15 & 6.2 \\
Total & 243 & 100.0 \\
\hline
\end{tabular}

Table 5 Patterns of ART switch of HIV/AIDS patients who changed their HAART regimen in east and west Wollega zone health institutions, April 28, 2007 to April 28, 2017

\begin{tabular}{lll}
\hline ART drugs after switching & Frequency & Percent \\
\hline AZT + 3TC + NVP & 47 & 19.3 \\
AZT + 3TC + EFV & 16 & 6.6 \\
TDF + 3TC + EFV & 56 & 23.05 \\
TDF + 3TC + NVP & 98 & 40.33 \\
ABC + ddi + LPV/R & 6 & 2.5 \\
AZT + 3TC + ATV/R & 6 & 2.5 \\
TDF + 3TC + LPV/R & 10 & 4.1 \\
TDF + 3TC + ATV/R & 4 & 1.6 \\
Total & 243 & 100.0 \\
\hline
\end{tabular}

health institutions which might have different co morbidities.

Majority of the study population 145 (59.67\%) had been on ART for $>10$ years. This finding indicated longer duration as compared to other studies as all patients stayed on ART for less than 3 years in Bedelle [15] and in Addis Ababa about $98 \%$ of patients stayed on ART for less than one and half years and in Dessie only $6 \%$ patients stayed on ART for more than 2 years $[10,16]$.

The main reason for initial ARV drug switch in the present study was toxicity which was known as peripheral neuropathy and it accounted for more than $60 \%$ of HAART regimen change. This finding was in agreement with the study conducted in some parts of Ethiopia [10]. The other reasons for HAART regimen change were hepatotoxicity 22(9.1\%), anemia $16(6.6 \%)$ and rash15 (5.3\%). The combined sum of hepatotoxicity, anemia and peripheral neuropathy was more than $80 \%$, which was much higher than the studies done in United Kingdom (35\%) [17] and India (27\%) [18]. However, it was almost similar to the studies conducted in other parts of Ethiopia as it was 75.8\% in Mekelle [19] and 66\% in Dessie [16]. But there was a significant heterogeneity on the type of toxicities in these studies.

Table 6 Common reasons for modification of regimens of study population in east and west Wollega zone health institutions, April 28, 2007 to April 28, 2017

\begin{tabular}{lll}
\hline Reason for regimen change & Frequency & Percent \\
\hline Peripheral neuropathy & 146 & 60.1 \\
Hepatotoxicity & 22 & 9.1 \\
d4t phase out & 18 & 7.4 \\
CNS toxicities & 16 & 6.6 \\
Anemia & 16 & 6.6 \\
Rash & 15 & 5.3 \\
Others & 10 & 4.9 \\
\hline
\end{tabular}


Table 7 Common reasons for modification by first treatment regimens among study population in east and west Wollega zone health institutions, April 28, 2007 to April 28, 2017

\begin{tabular}{|c|c|c|c|c|c|c|c|c|c|c|c|}
\hline \multirow{2}{*}{$\begin{array}{l}\text { Patterns } \\
\text { of ART Regimen }\end{array}$} & \multicolumn{10}{|c|}{ Reasons for ART regimen change } & \multirow[t]{2}{*}{ Tota } \\
\hline & Anemia & Rash & Peripheral neuropathy & Hepatotoxicity & Diarrhea & $\begin{array}{l}\text { CNS } \\
\text { toxicities }\end{array}$ & $\begin{array}{l}\text { stigma } \\
\text { disclosure }\end{array}$ & $\begin{array}{l}\mathrm{d} 4 \mathrm{t} \text { phase } \\
\text { out }\end{array}$ & jaundice & $\begin{array}{l}\text { Burning/ } \\
\text { numbness }\end{array}$ & \\
\hline d4t-3TC-NVP & 5 & 8 & 108 & 16 & 0 & 4 & 0 & 16 & 0 & 2 & 159 \\
\hline d4t-3TC-EFV & 0 & 3 & 30 & 6 & 0 & 10 & 2 & 2 & 0 & 2 & 55 \\
\hline AZT-3TC-NVP & 4 & 0 & 2 & 0 & 4 & 2 & 0 & 0 & 2 & 0 & 14 \\
\hline AZT-3TC-EFV & 7 & 2 & 6 & 0 & 0 & 0 & 0 & 0 & 0 & 0 & 15 \\
\hline Total & 16 & 13 & 146 & 22 & 4 & 16 & 2 & 18 & 2 & 4 & 243 \\
\hline
\end{tabular}

Change of the entire regimen from first-line to second-line is required in case of treatment failure. In order to increase likelihood of treatment success and minimize the risk of cross-resistance the new second-line regimen should involve drugs that keep activity against the patient's virus strain and should preferably include at least three new drugs, one or more from a new class [12]. The preferred strategy for second-line ART for adults is using a boosted PI and two NRTI combinations when NNRTI-containing regimens were used in first-line ART [8]. Patients from low-income countries were less likely to change two or more drugs and to change to a protease-inhibitor-containing regimen when compared with patients from high-income countries [20].

The study also found that there was high prevalence of TB as almost three out of five patients (57\%) had received isoniazid \& cotrimoxazole prophylaxis and more than half (52\%) of the patients had been treated for TB $[16,19]$. NVP has high interaction with Rifampicin which is strong liver enzyme (CYP 3A4) inducer where by therapeutic level of NVP is decreased up to $40 \%$ which necessitates switch to EFV. Additive hepatotoxicity effects also exists when NVP and Rifampicin were used together according to findings of some studies which is another requirement to switch from NVP to EFV as the latter has lesser adverse drug reaction with Rifampicin [21].

\section{Conclusions}

The number of patients who changed ARV drug in our resource constrained setting present a challenge to the restricted treatment choices that we currently own. The main reasons for ART switch were toxicity among which Peripheral neuropathy and hepatotocity were the leading toxicity for ART switch. Less toxic and better-tolerated HIV treatment options should be available and used more frequently in east and west Wollega zone health institutions. Patient should be evaluated regularly after a treatment change to assess for potential concerns with the new regimen, medication tolerance and to assess the effectiveness. Information is needed on patterns of resistance across the population to recommend future therapy options. Therefore national and health institution based surveillance of antiretroviral drug resistance should be conducted. It helps to know the resistance pattern and select the locally effective treatments. National level study on reasons for regimen change should be done to help drug suppliers and policy makers to improve and solve the problem. The reasons of ARV drug switch observed in this cross sectional study should be investigated further in longitudinal multicenter studies of ART utilization.

\section{Abbreviations}

3TC: Lamivudine; ABC: Abacavir; ART: Antiretro Viral Therapy; ARV: Antiretroviral; ATV/R: Atazanavir/Ritonavir; AZT: Zidovudine; CD4: Cluster of Difference; CNS: Central Nervous System; CYP: Cytochrome p-450; d4T: Stavudine; ddl: Didanosine; EFV: Efavirenz; FTC: Emitricibine; HAART: Highly Active Antiretroviral Therapy; LPV/R: Lopinavir/Ritonavir; NNRTI: Nucleoside Reverse Transcriptase inhibitor; NRTI: Nucleoside Reverse Transcriptase inhibitor; NVP: Nevirapine; Ol: Opportunistic Infection; PI: Protease Inhibitors; PLWHA: People Living With HIV/AIDS; SD: Standard deviation; TB : Tuberculosis; TDF: Tenofovir; VL: Viral Load; WHO: World Health Organization

\section{Acknowledgments}

The authors would like to express their sincere gratitude to Wollega University for their support for the accomplishment of this study. The authors are also thankful for officials of east and west Wollega zone health offices for delivering necessary information for this study. We would also like to thank supervisors and data collectors for taking their precious time to collect the data. We are also thankful for staff members of ART clinics from which the data was collected.

\section{Funding}

No funding was obtained for this study.

\section{Availability of data and materials}

All the necessary data were analyzed and included in this manuscript. Further data may be obtained from the primary author upon request.

\section{Authors' contributions}

The authors' responsibilities were as follows: $A B$ designed and supervised the study, and ensured quality of the data and made a substantial contribution to the local implementation of the study assisted in the analysis and interpretation of the data. BK participated in the design of the study, performed the data collection and the statistical analysis. GB also designed and supervised the study, and made a substantial contribution to the local implementation of the study. We want to ensure that all authors have performed all important points specified on criteria and guidelines for authorship and all authors read and approved the final manuscript. 


\section{Authors' information}

$A B$ is graduated from Jimma University with bachelor of pharmacy, from Unity University with bachelor of arts degree in economics and from Wollega University with masters of public health and has published many original research articles in an international journals, is currently working at Nekemte referral hospital chronic care pharmacy, P.O. Box 25, Nekemte, Ethiopia. BK is graduated from Wollega University with bachelor of pharmacy, is currently working at Nekemte referral hospital chronic care pharmacy, P.O. Box 25, Nekemte, Ethiopia. GB has also published many original research articles in an international journals, is currently a lecturer at Wollega University College of health and medical sciences, department of pharmacy, P.O. Box 395, Nekemte, Ethiopia.

\section{Ethics approval and consent to participate}

The study was approved by the ethical review committee of Wollega University. Official letter from the University explaining the aim of the study was submitted to zonal and woreda health offices of east and west Wollega zone and support of the administration was obtained prior to pursuing the study. Informed written consent was obtained from the study participants before data collection and clinical review of patients which was already recorded was used; confidentiality and anonymity of subject was maintained by not recording and identifying details, such as name or any other personal identifiers. No disclosure of any name of the patients, the healthcare provider or drug product was made in relation to the finding. Only numerical identifications were used as a reference. All data forms were stored in a confidential and secure place.

\section{Competing interests}

The authors declare that they have no competing interests.

\section{Publisher's Note}

Springer Nature remains neutral with regard to jurisdictional claims in published maps and institutional affiliations.

\section{Author details}

'Nekemte referral hospital, Nekemte, Ethiopia. ${ }^{2}$ Wollega University, Nekemte, Ethiopia.

Received: 15 September 2017 Accepted: 31 May 2018

Published online: 05 June 2018

\section{References}

1. Food, Medicine and Health Care Administration and Control Authority (FMHACA); Standard Treatment Guidelines for general hospitals; 3rd edition; Addis Ababa. Ethiopia 2014.

2. World Health organization (WHO); Fact Sheet; 2015

3. Central Statistical Agency Ethiopia and ICF International. Ethiopia demographic and health survey. Addis Ababa, Ethiopia and Calverton, Maryland: Central Statistical Agency and ICF International; 2012.

4. World Health Organization (WHO). HIV/AIDS Progress in 2014; Update Ethiopia, WHO country office for Ethiopia UNECA compound; Addis Ababa, Ethiopia; March 2015.

5. Federal democratic republic of Ethiopia. Country progress reports on HIV/ AIDS; Addis Ababa, Ethiopia; 2012.

6. Federal Ministry of Health $(\mathrm{MOH})$. Health Sector Development Program IV: (2010/11-2014/15), Final draft. Addis Ababa, Ethiopia: MOH. p. 2010.

7. Wilkin T, Marshall G, Gulick M. Switching antiretroviral therapy why, when and how. J Acquir Immune Defic Syndr. 2010;12:782-9.

8. World Health organization (WHO). Global update on HIV treatment: results, impact and opportunities, brief summary. Kuala Lumpur, Malaysia: WHO; $2013 a$.

9. Federal Ministry of Health $(\mathrm{MOH})$. Guideline for implantation of the antiretroviral therapy program in Ethiopia. Addis Ababa, Ethiopia: $\mathrm{MOH}$; 2007.

10. Jima T, Angamo M, Wabe N. Causes for antiretroviral regimen change among HIV/AIDS patients in Addis Ababa. Ethiopia Tanzania Journal of Health Research. 2013;15(1):1-9.

11. Park B, Choe $G$, Kim H, et al. Early modification of initial HAART regimen associated with poor clinical outcome in HIV patients. AIDS Res Hum Retrovir. 2010;23(8):794-800.
12. Hendrickson S, Jacobson P, Nelson W, et al. Host genetic influences on highly active antiretroviral therapy efficacy and AIDS free survival. J Acquire Immune Deficient Syndrome. 2008;48(3):263-71.

13. Demessie R, Mekonnen A, Amogne W, et al. Knowledge and adherence to antiretroviral therapy among adult people living with HIV / AIDS at TikurAnbessa specialized hospital, Ethiopia. International Journal of Basic \& Clinical Pharmacology. 2014;3(2):320-30.

14. Nekemte town health Office (NTHO) Census 2009.

15. Mekonnen Y, Molla G. Reason for regimen change among HIV patients on initial highly active antiretroviral therapy in Bedele. Journal of Biotechnology and Bio safety. 2014;2(4):116-22.

16. Mulugeta A, Chane T. Causes of antiretroviral drug changes among patients on antiretroviral therapy. Int J Pharm Sci Res. 2012;3(1):120-5.

17. Messou E, Anglaret X, Duvignac J, et al. Antiretroviral treatment changes in adults from Côte d'Ivoire: the roles of tuberculosis and pregnancy. AIDS (London, England). 2010;24(1):93-9.

18. Sandeep B, Vansant C, Raghunandan M, et al. Factors influencing the substitution of antiretroviral therapy in human immunodeficiency virus/ acquired immunodeficiency syndrome patients on first line highly active antiretroviral therapy. Asian Journal of Pharmaceutical and Clinical Research. 2014;7(5):117-20.

19. Bayou T, Woldu M, Gebre Meskel G, et al. Factors determinant for change of initial antiretroviral treatment regimen among patients on ART follow-up clinic of Mekelle hospital, Mekelle, Ethiopia. International Journal of Basic \& Clinical Pharmacology. 2014;3(1):44.

20. Zhou J, Kumarasamy N, Boyd M, et al. Deferred modification of antiretroviral regimen following documented treatment failure in Asia: Results from the TREAT Asia HIV Observational Database (TAHOD). HIV Medicine. 2010;11(1): $31-9$.

21. Hawkins C, Achenbach C, Fryda W, et al. Antiretroviral durability and tolerability in HIV-infected adults living in urban Kenya. J Acquir Immune Defic Syndr. 2009;45(3):304-10.

\section{Ready to submit your research? Choose BMC and benefit from:}

- fast, convenient online submission

- thorough peer review by experienced researchers in your field

- rapid publication on acceptance

- support for research data, including large and complex data types

- gold Open Access which fosters wider collaboration and increased citations

- maximum visibility for your research: over $100 \mathrm{M}$ website views per year

At BMC, research is always in progress.

Learn more biomedcentral.com/submissions 